\title{
A COMBINED CYCLOID FOOT TRAJECTORY AND VIRTUAL MODEL CONTROL FOR QUADRUPED ROBOTS
}

\author{
JINGYUAN SUN \\ Mechanical Engineering, National University of Singapore, 21 Lower Kent Ridge Road, \\ Singapore, 119077, Singapore \\ E-mail: sunj549@gmail.com \\ www.nus.edu
}

\begin{abstract}
In order to plan the foot trajectory and provide compliant behavior for quadruped robots, a locomotion controller based on the trajectory planning method and the virtual model is presented. The trajectory planning model is generated during the swing phase by using inverse kinematics and coordination transition. One method to reduce the fluctuation of speed and acceleration jumps is proposed here, generating fluent motion and avoiding slipping. In this paper, a virtual model controller has been built for the stance legs, providing computational simplification and highly compliant behavior. A quadruped robot platform in the simulation is used to test the proposed controller, both on the flat terrain and uneven terrain by using the trot gait. The simulation results show the effectiveness of the control algorithm, where the robot has the ability to generate smooth movement and overcome uneven terrain.
\end{abstract}

\section{Introduction}

Due to excellent environment adaptability and motion flexibility, quadruped robots have become a hot research direction in recent years. ${ }^{1,2}$ Many methods based on kinematic and dynamic methods have been developed.

Recent years have shown active research directions focusing on the development of quadruped robot systems and control methodologies. In 2010, HyQ has been developed by $\mathrm{IIT}^{3}$ based on hydraulic and motor drive. $\mathrm{ETH}^{4}$ proposed a quadruped robot StarlETH, with the advantage of smooth landing and resistance of lateral impact. MIT lab ${ }^{5}$ have developed an actuator system for their highly-dynamic robot Cheetah, which provides a high torque density and high-bandwidth force control. Except for the traditional legged robots, some designers have also made the combination of the legs and wheels for legged systems, which make the robots more flexible and versatile. ${ }^{6}$

The control task of quadruped robots is challenging due to the high degree of freedoms and the ground contact. ${ }^{7}$ There are many famous control methods that have been come out. One of the most representative concept is Central Pattern Generator (CPG). In [8], a controller is proposed to map the signals from CPG to walking trajectory in the workspace. Another direct method is the virtual model, proposed by Pratt. ${ }^{9}$ By simply using a set of virtual components, people could control the robot motion over level terrain. The virtual model is a widely used method since other algorithms could be developed based on it. ${ }^{10}$

The walking trajectory method is also useful and widely used since it could connect with other algorithms and models, such as CPG and virtual model. Many researchers have studied the walking trajectory method to design and plan the foot placement, which has good performance in fluent motion, arbitrary planning, and crossing obstacles. A periodic trajectory based on the wave motion can be found in [11], which could reduce the impact force contact. In [12], the relationship between energy consumption and gait parameters is demonstrated. The 2D sagittal plane could be much easier by avoiding dealing with the full complexity of 3D motion, ${ }^{13}$ and is widely used in the analysis of control tasks.

In contrast to previous approaches that only contain trajectory planning methods, ${ }^{14}$ 
a virtual model is included in our controller, which could provide compliant behavior for dynamic gaits. The structure of this paper is organized as follows. In the trajectory planning method, an improved foot placement is proposed during the swing phase. Through analyzing the kinematic model and coordinate transitions, the trajectories of the hip and knee joints are obtained. In the virtual model controller, the virtual forces exerted on the body is based on the optimal state and current state, and the feet contact force is mapped to the feedforward torques of the joints by using the transpose of the Jacobian matrix. The simulation has been done on both flat and uneven terrain to test the performance of the proposed method.

\section{Structure of Controller}

During one periodic motion of the quadruped robot, each leg proceeds with two phases: the swing phase and the stance phase. Here, in our controller, the foot follows a predefined cycloid curve during the swing phase and is controlled by the virtual model during the stance phase. The structure of the controller is shown in Fig. 1.

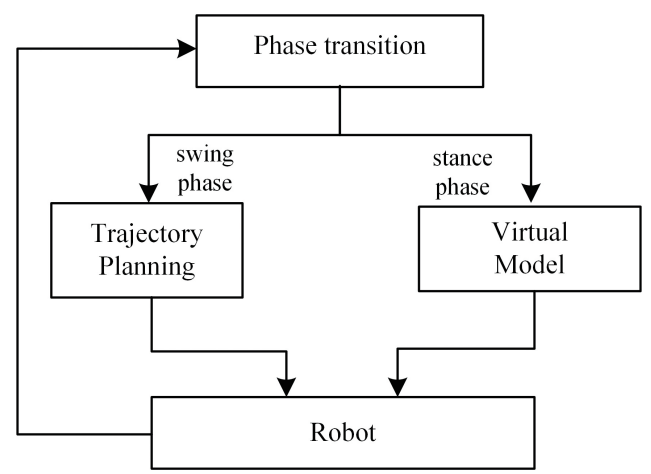

Fig. 1. The structure of the controller.

\section{Trajectory Planning}

The trajectory planning for the swing phase is presented in this section.

\subsection{Trajectory planning method}

The structure of the trajectory planning method is shown in Fig. 2. In the trajectory planning method, the foot placement $F(x, z)$ is generated during the swing phase, which could efficiently decrease the foot impact. Through the coordinate transition, the foot position ${ }_{F}^{o} F(x, z)$ under the body coordinate could be obtained. Then, by using the inverse kinematics model, the hip joint $\theta_{1}$ and the knee joint $\theta_{2}$ could be solved.

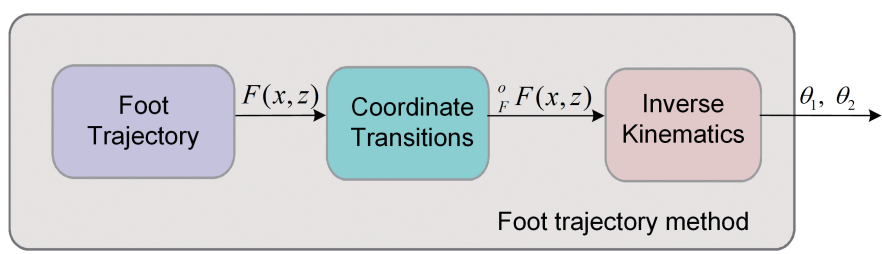

Fig. 2. The developed framework of Foot trajectory method. 


\subsection{Construction of coordinate}

In our quadruped model, each leg has two joints, which locate at the hip and knee. The world coordinate and the local coordinate of the robot are defined as $O x z$ and $O_{b} x_{b} z_{b}$. The coordinate of the body and the single leg is shown in Fig. 3(a). Here, the left front leg is chosen to declare the single leg $\mathrm{D}-\mathrm{H}$ coordinate, where the origin $O_{o} x_{o} z_{o}$ is located at the hip joint. $\theta_{1}$ and $\theta_{2}$ are the pitch angles of the hip and knee joints regarding to their balance positions $\theta_{h}$ and $\theta_{k}$, respectively. The position of the foot is defined by point $F(x, z)$. Here, for simplification, we only consider the pitch angle $\psi_{y}$ of the body.

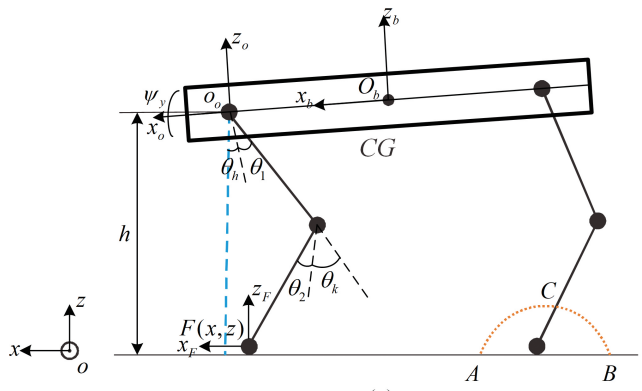

(a)

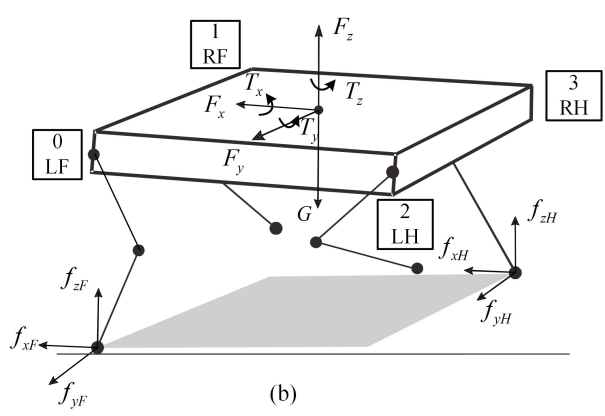

Fig. 3. (a) The developed framework of Foot trajectory method. (b) The static force model of the trot gait.

To establish the foot trajectory, the foot coordinate $O_{F} x_{F} z_{F}$ is built with respect to $O_{o} x_{o} z_{o}$, where $x_{F}$ has the same direction of the world coordinate $x$, and $z_{F}$ has the same direction of the world coordinate $z$. The coordinate transformation of $\left\{O_{F}\right\}$ to $\left\{O_{o}\right\}$ is :

$$
{ }_{F}^{o} T=\left[\begin{array}{cc}
I_{3} & o \\
E & P
\end{array}\right]
$$

where ${ }_{F}^{o} P=\left[\begin{array}{lll}0 & 0 & -h\end{array}\right]^{T}, h$ is the vertical dimension of the height from the hip joint to the ground, $I_{3}$ is a $3 \times 3$ identify matrix, $E=\left[\begin{array}{lll}0 & 0 & 0\end{array}\right]$. Assuming the body has a pitch angle of $\psi_{y}$, then the foot position $F(x, z)$ under $O_{o} x_{o} z_{o}$ is:

$$
{ }_{F}^{o} \mathrm{~F}=\left[\begin{array}{cc}
\operatorname{Rot}\left(y, \psi_{y}\right) & E^{T} \\
E & 1
\end{array}\right]{ }_{F}^{o} T^{F} P
$$

where ${ }^{F} P=\left[\begin{array}{lll}x_{F} & 0 & z_{F}\end{array}\right]^{T}$. If the pitch angle of the body $\psi_{y}=0$, then the foot position $F(x, z)$ under the single leg coordinate $O_{o} x_{o} z_{o}$ could be simplified as ${ }_{F}^{o} F=$ $\left[x_{F} 0 h-z_{F} 1\right]^{T}$.

\subsection{Foot trajectory planning}

In the swing phase, the foot leaves the ground and prepare for the next landing. The foot trajectory is planned under the coordinate of $O_{F} x_{F} z_{F}$. In the swing phase, [12] propose a composite cycloid trajectory, with the advantage of a reduction of the generation of inertia by swing the leg and reduction of body shaking. Here, in order to make the horizontal velocity become zero before the vertical velocity, the $x(t)$ has been divided into three segments. A stop time $T_{p}$ is set to keep the end point of the foot stays in position $x=-S_{o} / 2$, which is given by:

$$
T_{p}=\mu T_{y}
$$


where $\mu$ is a scale parameter from $(0,0.2]$. When $\mu$ is small, then the foot will stay at the position $x=-S_{o} / 2$ for a short time, while large $\mu$ will make the stop time become longer. The modified swing trajectory is defined as:

$$
\begin{gathered}
x_{\text {Fswing }}(t)=\left\{\begin{array}{cc}
-\frac{S_{o}}{2}, & 0 \leq t<T_{p} \\
S_{o}\left(\frac{t-T_{p}}{T_{y}-2 T_{p}}-\frac{1}{2 \pi} \sin \left(2 \pi \frac{t-T_{p}}{T_{y}-2 T_{p}}\right)\right)-\frac{S_{o}}{2}, T_{p} \leq t<T_{y}-T_{p} \\
\frac{S_{o}}{2}, & T_{y}-T_{p} \leq t<T_{y}
\end{array}\right. \\
z_{\text {Fswing }}(t)=\left\{\begin{array}{cc}
2 H_{o}\left(\frac{t}{T_{y}}-\frac{1}{4 \pi} \sin \left(4 \pi \frac{t}{T_{y}}\right)\right), & 0 \leq t<\frac{T_{y}}{2} \\
2 H_{o}\left(1-\frac{t}{T_{y}}+\frac{1}{4 \pi} \sin \left(4 \pi \frac{t}{T_{y}}\right)\right), & \frac{T_{y}}{2} \leq t<T_{y}
\end{array}\right.
\end{gathered}
$$

where $S_{o}$ is the step length (stride length), $T_{y}$ is the swing phase period, $H_{o}$ is the maximum foot height. Here, set the step length $S_{o}=200 \mathrm{~mm}$, maximum foot height $H_{o}=50 \mathrm{~mm}$, the swing phase period $T_{y}=0.5 \mathrm{~s}$, and the scale parameter $\mu=1 / 20$. The acceleration and velocity on $x$ axis are 0 at the time of $t \in\left[0,1 / 20 T_{y}\right)$ and $t \in\left[19 / 20 T_{y}, T_{y}\right)$, while the acceleration and velocity on $z$ axis are 0 at the time of $t=0, t=T_{y} / 2$ and $t=T_{y}$. This trajectory could reduce the impact against the floor. The single leg foot trajectory of swing phase is shown in Fig. 4.

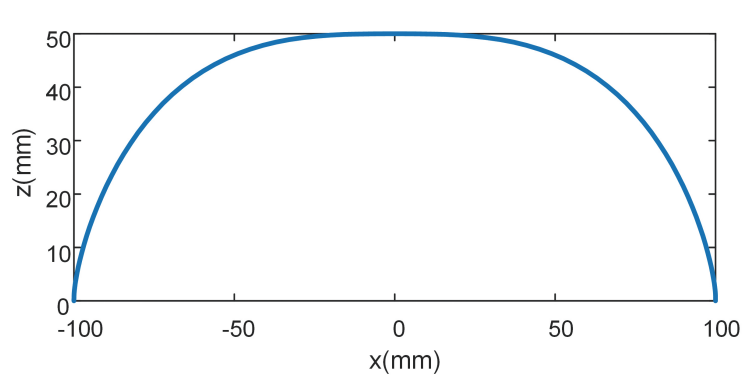

Fig. 4. The foot trajectory of single leg.

\subsection{Kinematic Analysis}

Since the foot trajectory and the coordinate of the single leg have been built, the foot trajectory under the hip coordinate $O_{o} x_{o} z_{o}$ could be calculated in the swing phase, by using the coordinate transitions Eq. (2). Where $x_{F}$ and $z_{F}$ is the coordinate of $x$ axis and $z$ axis of the foot trajectory.

Under the single leg coordinate $O_{o} x_{o} z_{o}$, the hip pitch joint $\theta_{1}$ and the knee pitch joint $\theta_{2}$ to the point $F(x, z)$ could be obtained by solving the inverse kinematics equation:

$$
\begin{array}{r}
\theta_{2}=\varepsilon \arcsin \frac{x^{2}+z^{2}-l_{1}{ }^{2}-l_{2}{ }^{2}}{2 l_{1} l_{2}}-\theta_{h} \\
\theta_{1}=\arctan 2\left(s_{1}, c_{1}\right)
\end{array}
$$

where

$$
\begin{gathered}
c_{1}=\frac{\varepsilon l_{2} c_{2} x-\left(l_{1}+l_{2} s_{2}\right) z}{x^{2}+z^{2}} \\
s_{1}=\frac{-\varepsilon\left(l_{1}+l_{2} s_{2}\right) x-l_{2} c_{2} z}{x^{2}+z^{2}}
\end{gathered}
$$

$\theta_{h}$ are the balance position angles of the hip joints, $\theta_{1}$ and $\theta_{2}$ are the pitch angles of the hip and knee joints, respectively. Here, we set the length of the thigh leg and shin leg 
$l_{1}=l_{2}=350 \mathrm{~mm}$, the height from the hip joint to the ground $h=600 \mathrm{~mm}$, the balance position angles $\theta_{h}=30^{\circ}$ for the front legs, $\theta_{h}=-30^{\circ}$ for the hind legs, and $\varepsilon$ :

$$
\varepsilon=\left\{\begin{array}{cc}
1, & \text { Front leg } \\
-1, & \text { Hind leg }
\end{array}\right.
$$

\section{Virtual Model Controller}

In this section, a virtual model controller is presented for the stance legs, based on [9], providing computational simplification and highly compliant behavior.

\subsection{Joint Torques}

Set $\{0123\}$ to describe the number of the four legs $\{\mathrm{LF}, \mathrm{RF}, \mathrm{LH}, \mathrm{RH}\}$, representing the left front, right front, right hind and left hind leg separately. Define $u$ and $v$ to represent the number of front legs and hind legs, respectively. For the trot gait, the set of $u$ and $v$ is always picked up from diagonal pairs: (i) $u=0, v=3$ (ii) $u=1, v=2$. For the pace gait, the set of $u$ and $v$ is always picked up from ipsilateral pairs: (i) $u=0, v=2$ (ii) $u=1$, $v=3$.

The foot contact force is expressed as $\vec{f}_{\text {foot_i }}=\left[f_{x i} f_{y i} f_{z i}\right]^{T}$, where the subscript $i=u, v$ is used to represent the number of the stance legs, and $u$ and $v$ could be picked up from the previously defined set for different gaits. In dynamic gaits, the front and hind stance legs receive the ground contact force $\vec{f}_{\text {foot_u } u}$ and $\vec{f}_{\text {foot_v }}$. The trunk is subjected to gravity. Decompose these three forces along the body coordinate $x_{b}, y_{b}$, and $z_{b}$, as shown in Fig. 3(b). Here, for simplification, we ignore the inertia of the robot's legs. The virtual force exerted on the trunk could be represented as:

$$
\begin{array}{r}
F_{x}=\sum f_{x i}+G_{x} \\
F_{z}=\sum f_{z i}+G_{z} \\
T_{x}=\sum f_{z i} y_{i}-\sum f_{y i} z_{i} \\
T_{y}=\sum f_{x i} z_{i}-\sum f_{z i} x_{i} \\
T_{z}=\sum f_{y i} x_{i}-\sum f_{x i} y_{i}
\end{array}
$$

where $\left\{x_{i}, y_{i}, z_{i}\right\}$ are the coordinates of $i$ th stance legs under the body reference frame $O_{b} x_{b} z_{b}$, and the subscript $i=u, v$ is picked up from the previously defined set for different gaits. Here, five equations are along with six unknowns. For the sixth constraint, we simply choose it to match the $y$-axis feet contact forces, $f_{y u}=f_{y v}$. The above equations could be simplified as:

$$
\vec{F}=Q \vec{f}_{\text {foot }}
$$

where

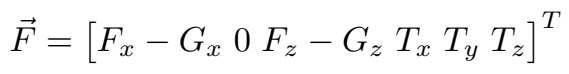

$$
\begin{aligned}
& Q=\left[\begin{array}{ll}
I_{3} & E_{H} \\
R_{u} & R_{v}
\end{array}\right] \\
& R_{i}=\left[\begin{array}{ccc}
0 & -z_{i} & y_{i} \\
z_{i} & 0 & -x_{i} \\
-y_{i} & x_{i} & 0
\end{array}\right], i=u, v
\end{aligned}
$$




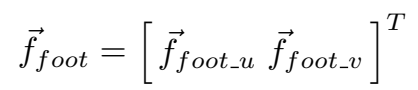

$\vec{f}_{\text {foot }}$ is the feet contact force of the front and hind stance legs, $Q$ is a $6 \times 6$ matrix, $I_{3}$ is a $3 \times 3$ identify matrix, $E_{H}=\operatorname{diag}(1,-1,1), R_{u}$ and $R_{v}$ represent the $3 \times 3$ skew-symmetric matrix of the front and hind legs, respectively. The gravity forces $\left[G_{x} G_{z}\right]^{T}$ is approximated by using the pitch angle $\psi_{y}$ of the CoM:

$$
\left[\begin{array}{l}
G_{x} \\
G_{z}
\end{array}\right]=\left[\begin{array}{c}
M g \sin \left(\psi_{y}\right) \\
-M g \cos \left(\psi_{y}\right)
\end{array}\right]
$$

We could control the body motion of the robot by adjusting the virtual force, and we will give the detail in the next part. Then, the feet contact force could be calculated by using the inverse matrix of $Q$ and $\vec{F}: \vec{f}_{\text {foot }}=Q^{-1} \vec{F}$. After that, the feet contact force could be mapped to the feedforward torques of the joints $\vec{\tau}$ for the stance legs by using the transpose Jacobian matrix of the supporting legs :

$$
\vec{\tau}=\left[\begin{array}{cc}
J_{u}{ }^{T} & 0 \\
0 & J_{v}^{T}
\end{array}\right] \vec{f}_{\text {foot }}
$$

\subsection{The body motion control}

By controlling the virtual force on the trunk, we could realize the body motion control of the robot. The virtual force exerted on the CoM of the body is based on the optimal state and the current state. Since $y$-axis control is seldom directly used in natural quadruped animals, we omit the $y$-axis virtual force control here. We use a virtual spring-damper model to adjust the row pitch yaw (RPY) angle, height, and velocity of the torso:

$$
\left[\begin{array}{c}
F_{x} \\
F_{z} \\
T_{x} \\
T_{y} \\
T_{z}
\end{array}\right]=\left[\begin{array}{c}
K_{v x}\left(v_{x d}-v_{x}\right) \\
K_{h}\left(h_{b d}-h_{b}\right)+K_{v z}\left(v_{z d}-v_{z}\right) \\
K_{\phi x}\left(\phi_{x d}-\phi_{x}\right)+K_{\omega x}\left(\omega_{x d}-\omega_{x}\right) \\
K_{\psi y}\left(\psi_{y d}-\psi_{y}\right)+K_{\omega y}\left(\omega_{y d}-\omega_{y}\right) \\
K_{\omega z}\left(\omega_{z d}-\omega_{z}\right)
\end{array}\right]
$$

where $\boldsymbol{K}$ is the stiffness of the spring and damper model, $v_{x d}, h_{b d}, v_{z d}, \phi_{x d}, \omega_{x d}, \psi_{y d}, \omega_{y d}$ and $\omega_{z d}$ are the desired velocity, $z$-axis height, $z$-axis speed, roll angle, $x$-axis angular velocity, pitch angle, $y$-axis angular velocity, and $z$-axis velocity, respectively. The body height $h_{b}$ is calculated by averaging the height of the front and hind stance leg $h_{b}=-\left(z_{F}+z_{H}\right) / 2$.

\section{Simulation}

To validate our controller, we demonstrate trot gait on flat terrain and uneven terrain in the simulation platform, Webots. The specifications of the quadruped model are shown in Table 1. In the simulation, the frequency is set to $200 \mathrm{~Hz}$. At each cycle, IMU receives the acceleration $\vec{a}$ and angular velocity $\vec{\omega}$ of the body, and each position sensors on the joints receive the data of the motor positions. Here, in order to model the uneven terrain, we use an Uneven Terrain Proto to create noisy elevation grids based on a Perlin noise, with few parameters. In the elevation grids, the dimension of $z$ axis is $0.2 \mathrm{~m}$. The snapshots are shown in Fig. 5.

In both experiments, the swing legs of the robot are controlled by the trajectory planning method and the stance legs are controlled by the virtual model. We compare our controller with the other two methods: (i) using the trajectory planning method in [14] for both swing legs and stance legs (ii) using the virtual model for stance legs and using fixed values for swing legs in [15]. 
Table 1. Parameters of the quadruped model.

\begin{tabular}{ccc}
\hline Parameter & Definition & Value \\
\hline $\mathrm{b}$ & Half of the body length & $0.5 \mathrm{~m}$ \\
$\mathrm{a}$ & Half of the body width & $0.25 \mathrm{~m}$ \\
$\mathrm{~h}$ & Body height & $0.2 \mathrm{~m}$ \\
$l_{1}$ & Length of the leg thigh & $0.35 \mathrm{~m}$ \\
$l_{2}$ & Length of the leg shin & $0.35 \mathrm{~m}$ \\
$m_{b}$ & Mass of the body & $55 \mathrm{~kg}$ \\
$m_{t}$ & Mass of the leg thigh & $7 \mathrm{~kg}$ \\
$m_{s}$ & Mass of the leg shin & $2 \mathrm{~kg}$ \\
\hline
\end{tabular}

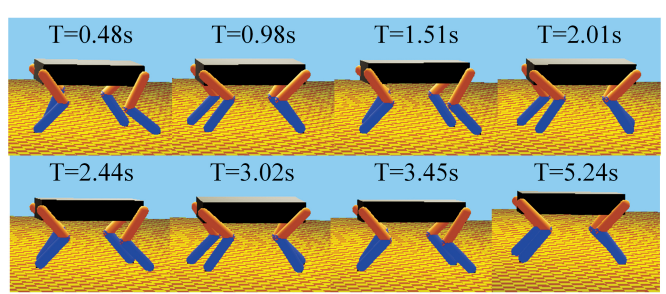

(a)

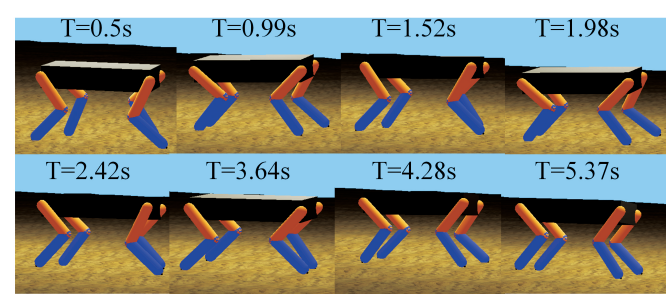

(b)

Fig. 5. Simulation capture in Webots. (a) Trot gait on the flat terrain. (b) Trot gait on the uneven terrain.

In the first simulation, the robot trots on the flat terrain. As shown in Fig.6(a), our controller has advantages in terms of body velocity and orientation. Compared to the method (i), our controller has fewer roll fluctuations and a larger forward speed. Compared to the method (ii), our controller has an apparent improvement of the forward speed when orientation fluctuations are similar.

In the second experiment, our controller demonstrates the ability to overcome uneven terrain. During the movement, the robot could tolerate some slippage when the swing angles are not large enough to cause instability. We linearly increase the dimension of the uneven terrain in $z$ axis by $0.02 \mathrm{~m}$, from $0.2 \mathrm{~m}$ to $0.3 \mathrm{~m}$. The robot gets easier slip when the $z$ dimension is more than $0.24 \mathrm{~m}$. The body orientation and linear velocity for this task are shown in Fig.6(b). Compared to flat terrain movement, the roll angle of the body has a larger fluctuation. This is because the touchdown feet has an apparent difference of the height on the uneven terrain compared to flat terrain, causing a larger roll angle. Compared to the method (i), our controller has a small advantage of forward speed. Compared to the method (ii), our controller has a larger forward speed and could keep balance.

\section{Conclusion}

In this paper, we have presented a locomotion controller consisting of a trajectory planning method and a virtual model. An improved trajectory during the swing phase has been built, where the horizontal velocity becomes zero before the vertical velocity, with low impact and smooth movement. The virtual model controller provides computational simplification and highly compliant behavior, where the robot has the ability to overcome uneven terrain.

In future, we aim to develop the online force feedback to detect the contact condition of the swing-leg. We also aim to update the phase transition machine by receiving more data, such as sensory feedback to the model. 

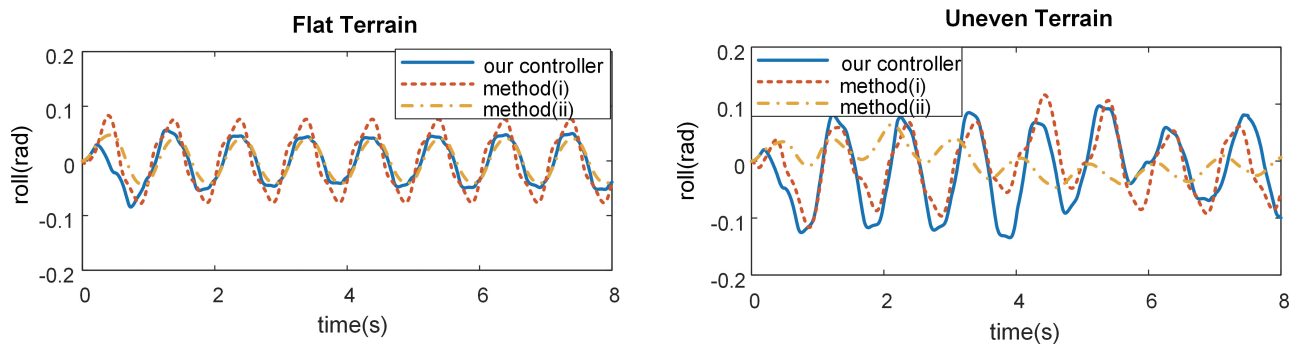

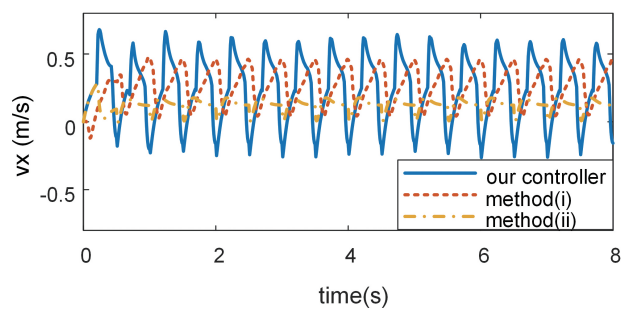

(a)

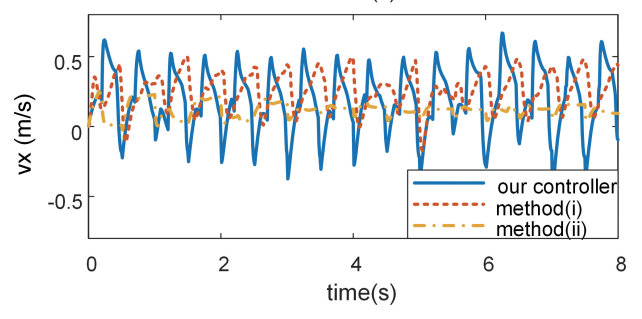

(b)

Fig. 6. Plots of body orientation and linear velocity. (a) Trot gait on the flat terrain. (b)Trot gait on the uneven terrain.

\section{References}

1. M. Bjelonic, C. D. Bellicoso, Y. de Viragh, D. Sako, F. D. Tresoldi, F. Jenelten and M. Hutter, IEEE Robotics and Automation Letters (2019).

2. C. D. Bellicoso, F. Jenelten, C. Gehring and M. Hutter, IEEE Robotics and Automation Letters 3, 2261 (2018).

3. C. Semini, V. Barasuol, T. Boaventura, M. Frigerio, M. Focchi, D. G. Caldwell and J. Buchli, The International Journal of Robotics Research 34, 1003 (2015).

4. M. Hutter, C. Gehring, M. Bloesch, M. A. Hoepflinger, C. D. Remy and R. Siegwart, Starleth: A compliant quadrupedal robot for fast, efficient, and versatile locomotion, in Adaptive Mobile Robotics, (World Scientific, 2012) pp. 483-490.

5. G. Bledt, M. J. Powell, B. Katz, J. Di Carlo, P. M. Wensing and S. Kim, Mit cheetah 3: Design and control of a robust, dynamic quadruped robot, in 2018 IEEE/RSJ International Conference on Intelligent Robots and Systems (IROS), 2018.

6. M. Geilinger, R. Poranne, R. Desai, B. Thomaszewski and S. Coros, ACM Transactions on Graphics (TOG) 37, p. 160 (2018).

7. M. Neunert, F. Farshidian, A. W. Winkler and J. Buchli, IEEE Robotics and Automation Letters 2, 1502(July 2017).

8. C. Liu, Q. Chen and D. Wang, IEEE Transactions on Systems, Man, and Cybernetics, Part B (Cybernetics) 41, 867(June 2011).

9. J. Pratt, C.-M. Chew, A. Torres, P. Dilworth and G. Pratt, The International Journal of Robotics Research 20, 129 (2001).

10. C. Mastalli, I. Havoutis, M. Focchi, D. Caldwell and C. Semini (2018).

11. Y. Sakakibara, K. Kan, Y. Hosoda, M. Hattori and M. Fujie, Foot trajectory for a quadruped walking machine, in EEE International Workshop on Intelligent Robots and Systems, Towards a New Frontier of Applications, 1990.

12. Lei, Jingtao, Wang, Feng, Yu, Huangying, Wang, Tianmiao, Yuan and Peijiang, Chinese Journal of Mechanical Engineering 27, 138 (2014).

13. S. Coros, A. Karpathy, B. Jones, L. Reveret and M. van de Panne, ACM Trans. Graph. 30, 59:1(July 2011).

14. Y. Zeng, J. Li, S. Yang and E. Ren, Applied Sciences 8, p. 56 (2018).

15. C. P. Santos and V. Matos, Robotics and Autonomous Systems 59, 620 (2011). 